\title{
Performance of unanchored matching- adjusted indirect comparison (MAIC) for the evidence synthesis of single-arm trials with time-to-event outcomes
}

Yawen Jiang ${ }^{1 *}$ (D) and Weiyi $\mathrm{Ni}^{2}$

\begin{abstract}
Background: The objectives of the present study were to evaluate the performance of a time-to-event data reconstruction method, to assess the bias and efficiency of unanchored matching-adjusted indirect comparison (MAIC) methods for the analysis of time-to-event outcomes, and to propose an approach to adjust the bias of unanchored MAIC when omitted confounders across trials may exist.

Methods: To evaluate the methods using a Monte Carlo approach, a thousand repetitions of simulated data sets were generated for two single-arm trials. In each repetition, researchers were assumed to have access to individuallevel patient data (IPD) for one of the trials and the published Kaplan-Meier curve of another. First, we compared the raw data and the reconstructed IPD using Cox regressions to determine the performance of the data reconstruction method. Then, we evaluated alternative unanchored MAIC strategies with varying completeness of covariates for matching in terms of bias, efficiency, and confidence interval coverage. Finally, we proposed a bias factor-adjusted approach to gauge the true effects when unanchored MAIC estimates might be biased due to omitted variables.
\end{abstract}

Results: Reconstructed data sufficiently represented raw data in the sense that the difference between the raw and reconstructed data was not statistically significant over the one thousand repetitions. Also, the bias of unanchored MAIC estimates ranged from minimal to substantial as the set of covariates became less complete. More, the confidence interval estimates of unanchored MAIC were suboptimal even using the complete set of covariates. Finally, the bias factor-adjusted method we proposed substantially reduced omitted variable bias.

Conclusions: Unanchored MAIC should be used to analyze time-to-event outcomes with caution. The bias factor may be used to gauge the true treatment effect.

Keywords: Matching-adjusted, Indirect comparison, Reconstruction: single-arm, Evidence synthesis

\footnotetext{
* Correspondence: jiangyw26@mail.sysu.edu.cn

'School of Public Health (Shenzhen), Sun Yat-sen University, Room 215,

Mingde Garden \#6, 132 East Outer Ring Road, Pan-yu District, Guangzhou, Guangdong, China

Full list of author information is available at the end of the article
}

(c) The Author(s). 2020 Open Access This article is licensed under a Creative Commons Attribution 4.0 International License, which permits use, sharing, adaptation, distribution and reproduction in any medium or format, as long as you give appropriate credit to the original author(s) and the source, provide a link to the Creative Commons licence, and indicate if changes were made. The images or other third party material in this article are included in the article's Creative Commons licence, unless indicated otherwise in a credit line to the material. If material is not included in the article's Creative Commons licence and your intended use is not permitted by statutory regulation or exceeds the permitted use, you will need to obtain permission directly from the copyright holder. To view a copy of this licence, visit http://creativecommons.org/licenses/by/4.0/ The Creative Commons Public Domain Dedication waiver (http://creativecommons.org/publicdomain/zero/1.0/) applies to the data made available in this article, unless otherwise stated in a credit line to the data. 


\section{Introduction}

Comparative effectiveness evidence is essential for clinical decision and formulary policy making, heath technology assessments, and economic evaluations. When direct comparisons and network meta-analyses (NMA) are infeasible, population-adjusted indirect comparison methods may be used for evidence syntheses of comparative effectiveness [1]. Such methods include matching-adjusted indirect comparison (MAIC), simulated treatment comparisons (STC), and multi-level network meta regression (MLNMR) [1, 2], among which MAIC is relatively popular $[1,3]$. The process of conducting an MAIC has been described extensively in a number of previous studies [3-5]. At its core, the MAIC method utilizes the individual-level patient data (IPD) from the trial of an intervention (usually a manufacturer's own product) and the published aggregate data from the trial of a comparator intervention, and re-weights the patients with IPD such that their characteristics are balanced with those of the patients from the aggregate data of the comparator's trial [3]. The weights can be obtained using propensity scores estimated with method of moments or entropy balancing, either of which is calculated using the observed characteristics that need to be balanced $[3,5]$. The outcome of the patients with IPD calculated with re-weighting is then compared with that of the published aggregate data to obtain the relative effect [3].

MAIC has received increasing popularity in the evidence-based medicine community and health technology assessment agencies $[1,6,7]$. It has been mostly implemented in an anchored approach in which a common comparator, such as the placebo group, is available across trials [6]. The relative effect in the analyses of time-to-event outcomes or survival analyses in anchored MAIC, usually quantified as hazard ratio (HR), is calculated by taking the ratio of HRs from different trials or the difference of logHRs [8]. Because of the common comparator, anchored MAIC estimates are theoretically not biased by the existence of unbalanced prognostic variables that are not effect modifiers [6]. In the less frequently used unanchored MAIC approach, a common comparator is not available and the outcomes from the re-weighted IPD and the published aggregate data must be compared directly. Hence, a key difference of unanchored MAIC from anchored MAIC is that the former compares outcomes across trials whereas the latter conceptually compares treatment effect across trials. However, at least two additional complexities arise in the unanchored analyses of time-to-event outcomes that may potentially nullify the properties of anchored MAIC. First, unbalanced prognostic variables can themselves contribute to the outcome and may become confounders without adjustment. Realizing such potential drawbacks, Phillipo et al. recommended that unanchored MAIC was not always advisable [1]. Second, HRs are estimated using regression techniques such as Cox regression that requires the IPD of the published study instead of the published aggregate data, which distinguished itself from unanchored MAIC for linear-scale outcomes in which the aggregate outcomes were compared after reweighting. Such data is typically not available to researchers and is obtained through reconstruction of digitized Kaplan-Meier (K-M) curves [9, 10]. For ease of distinction, we refer to the reconstructed IPD using digitized $\mathrm{K}-\mathrm{M}$ curves as RIKM hereinafter.

Previous discussion of and studies on the properties of MAIC have focused on anchored analyses of linear-scale outcomes [3-6]. The properties of unanchored MAIC in the context of time-to-event analysis have not been investigated so far yet the literature is gradually picking up the approach without appreciating the unique profiles of this estimator [11-13]. This represents a major methodological gap that needs to be filled. Specifically, single-arm trials accounted for $50 \%$ of all US Food and Drug Administration (USFDA) accelerated hematology and oncology approvals in 2015 , which continued to surge to $80 \%$ in 2018 [14]. In light of this, it is expected that more comparative effectiveness studies and economic evaluations have to utilize unanchored MAIC on time-to-event outcomes at the absence of common comparators. However, due to the two aforementioned complexities, several questions that are related to the properties of unanchored MAIC on time-to-event outcomes remain to be answered. First, does RIKM represent the original survival data well enough? This is the premise that unanchored MAIC based on RIKM can be used for indirect comparison. Although this has been partially addressed when the data reconstruction method was originally proposed, the validation of data reproducibility was only conducted by comparing the summary measures of survival data underlying one single graph to the summary measures of the reconstructed version of the same graph [9]. Surprisingly, there has been an absence of attempt to validate the reconstruction method using simulation, which was likely due to the requirement of labor-intensive manual operation. Specifically, such a simulation analysis involves digitizing the curve of each repetition that mandates manually defining the coordinates, identifying the curve, and exporting the data. This process is unlike typical simulation studies that can be fully automated with programming. Hence, a simulation-based evaluation of the performance of the reconstruction approach is needed to verify its utility. Second, what are the properties of unanchored MAIC on time-to-event outcomes with respect to bias and efficiency in different scenarios? For example, is it unbiased if all prognostic variables are captured in the creation of weight as in the case of linear outcomes regardless of fundamentally different statistical processes? Also, is it unbiased if prognostic factors and effect modifiers are unbalanced 
across trials? A simulation study may be effective to reveal its performance in such scenarios. Third, is there a statistical approach to estimate the boundary of the true effect if unanchored MAIC estimates are indeed biased by unbalanced and unobserved covariates? We examined if the concept of bias factor that is borrowed from observational cohort studies can be used for this purpose. To answer such questions, we conducted a simulation study to investigate the properties of unanchored MAIC on time-to-event outcomes in different scenarios. The results can shed light on the above-mentioned issues and help to guide the appropriate use of the unanchored MAIC on time-to-event outcomes.

\section{Methods}

\section{Simulated data}

Two scenarios were simulated to investigate the properties of unanchored MAIC on time-to-event outcomes. Under each scenario, hypothetical data of breast cancer patients were simulated for two single-arm trials. For simplicity, the interventions in the trials were called treatment $\mathrm{A}$ and $\mathrm{B}$, respectively. It was assumed that researchers had access to the IPD of treatment A but not to that of comparator B. The purpose of unanchored MAIC was, therefore, to compare the effectiveness of $B$ versus $A$. The outcome in the trials was recurrence-free survival time (RFS), which was defined as the time from the start of the intervention to the earlier of all-cause death and disease recurrence. In both scenarios, unbalanced covariates across trials were simulated by design. In the present study, an effect modifier was defined as a variable that interacted with the intervention in the data generation of time to event and a prognostic factor was a variable that itself loaded on time to event. In the first scenario, effect modifiers of treatments were not included. The same set of prognostic factors were simulated for each arm (or trial), which were age, an indicator variable for menopausal status (postmenopausal vs. not), and indicator variables for tumor grades $(1,2$, and 3$)$. The $B$ arm data also contained an indicator of treatment B. The estimated effect of B in relation to A using unanchored MAIC was captured by the HR associated with this indicator. The prognostic factors of the A arm were set so such that the patients in the A arm were in less severe conditions than the B arm. In other words, the A arm had lower average values of the prognostic factors that were negatively associated with RFS. Age was a continuous variable and was simulated using normal distributions truncated at the lower and upper bounds, while the other prognostic factors were indicators simulated using Bernoulli distributions. The specifications of the distributions used for the prognostic factors of the two arms are listed in Table 1. The sample sizes of the A and B arms were arbitrarily set at 1000 and 800 , respectively.

The RFS were simulated using Weibull distributions. The shape and scale parameters of the Weibull

Table 1 Parameters used in the simulation of the $A$ and $B$ arms

\begin{tabular}{lll}
\hline & A arm & B arm \\
\hline Sample size & 1000 & 800 \\
Mean age & 53 & 56 \\
Standard deviation of age & 11 & 12 \\
Range of age & {$[21,80]$} & {$[23,85]$} \\
Menopausal status (\% post-menopausal) & 40 & 50 \\
tumor grade =1 (\%) & 20 & 10 \\
tumor grade =2 (\%) & 65 & 70 \\
tumor grade =3 (\%) & 15 & 20 \\
Distribution & Weibull & Weibull \\
Scale parameter & 0.00000004 & 0.00000004 \\
Shape parameter & 2.2 & 2.2 \\
Coefficients of variables (logHR) & & \\
Age (yearly increment) & 0.02 & 0.02 \\
Menopausal status & 0.5 & 0.5 \\
Indicator variable for tumor grade = 2 & 0.3 & 0.3 \\
Indicator variable for tumor grade = 3 & 0.6 & 0.6 \\
B indicator & NA & -0.5 (in the scenario without effect modifier) / -0.4 (in the scenario with effect modifier) \\
B indicator $\times$ menopausal status interaction & NA & -0.2 (only applicable to the scenario with effect modifier) \\
Maximum follow-up time (days) & 2500 & 2500 \\
\hline Ab & &
\end{tabular}

Abbreviation: HR Hazard ratio; NA Not applicable 
distribution and the coefficients of the prognostic factors in the linear component of the Weibull distribution are displayed in Table 1. Random censoring was included based on a uniform distribution that was truncated at 2500 days. In the second scenario, not only the prognostic factors were unbalanced but also menopausal status was both a prognostic factor and an effect modifier of $B$. There was no change to the A arm. Hence, the A arm was directly taken from the simulation in scenario 1 . Whereas the coefficients of the other prognostic factors remained the same in the B arm across the scenarios, that of the B indicator was changed from -0.5 in the first scenario to -0.4 in the second scenario. In addition, an interaction term of the B indicator and menopausal status was included in the linear component to incorporate the modification effect. The coefficient of the interaction term was set at -0.2 such that the expected treatment effect was the same across the two scenarios. The simulated data in the B arms were used to generate aggregate characteristics and K-M curves, which correspond to the published data of typical single-arm clinical trials. One-thousand sets of triplet timeto-event data (one for the $\mathrm{A}$ arm, one for the $\mathrm{B}$ arm without any effect modifiers, and one for the B arm with an effect modifier) were simulated. Subsequent analyses of the statistical performance of unanchored MAIC using different analytic strategies were conducted between the $\mathrm{A}$ arm and the B arms within each set. The results from the 1000 repetitions formed the distributions of the estimates using alternative MAIC strategies which are described later. As mentioned previously, each repetition in the present study involved digitizing the hypothetically published K-M curve of the B arm and required heavy manual operation. Hence, the number of repetitions was restricted to 1000 .

\section{Validation of digitization-based reconstruction method}

The validation of the reconstruction method was based on comparison of the 1000 repetitions of RIKM and the simulated data for the generation of the curves. RIKM of both B arms (with and without the effect modifier) were compared to the corresponding simulated raw data using Cox regressions with an indicator of being reconstructed data and a variable representing the time-varying effect of the "being reconstructed" indicator. The hypotheses were that the HRs of the indicator and the time-varying effect would both equal one if the reconstructed data sufficiently mirrored the raw data. To quantify the assessment, the mean HRs were estimated as

$$
\overline{\mathrm{HR}}_{\mathrm{k}}^{r c}=\frac{1}{\mathrm{~N}} \sum_{j=1}^{N}\left(\widehat{\mathrm{HR}_{\mathrm{j}, \mathrm{k}}^{r c}}\right)
$$

$$
\overline{\mathrm{HR}}_{\mathrm{k}}^{t v}=\frac{1}{\mathrm{~N}} \sum_{j=1}^{N}\left(\widehat{\mathrm{HR}_{\mathrm{j}, \mathrm{k}}^{t v}}\right)
$$

where $\overline{\mathrm{HR}}_{\mathrm{k}}^{r c}$ and $\overline{\mathrm{HR}}_{\mathrm{k}}^{t v}$ are respectively the mean HRs of the reconstruction indicator and the time-varying effect, $\mathrm{N}$ is the number of repetitions in each scenario, and $\widehat{\mathrm{HR}_{\mathrm{j}, \mathrm{k}}^{r c}}$ and $\widehat{\mathrm{HR}_{\mathrm{j}, \mathrm{k}}^{t v}}$ are respectively the estimated HRs of the reconstruction indicator and the time-varying effect from the jth repetition of the kth (1st or 2nd) scenario. Also, the percentages of the $95 \%$ confidence intervals (CIs) that covered one were calculated for both estimates in both scenarios. It was expected that the percentages were at least $95 \%$.

\section{Strategies of unanchored MAIC on time-to-event outcomes}

The general data analytic steps of unanchored MAIC are 1) balancing the IPD with the aggregate data to obtain weights; 2) digitization for RIKM; and 3) pooling the IPD and the RIKM to conduct weighted survival analysis. Two methods have been proposed to balance the prognostic factors of the IPD data with those of aggregate data, namely propensity score matching using a method-of-moments logistic regression and entropy balancing $[1,15]$. Phillipo et al. noticed that the two methods are equivalent in reducing bias yet the latter generates smaller standard errors [1]. Also, entropy balancing generates equal weighted sample sizes of the two groups [16]. In the present study, entropy balancing was used to balance the prognostic factors and to obtain weights. Both the mean and the variance of age were used for balancing. This reflects real-world practice because the variance of characteristics such as that of age is usually reported in the publication of clinical trials. The other covariates including the indicator of menopausal status and the indicators of tumor grades were balanced on the percentages. These variables were dichotomous variables of which the balance of the second moment follows that of the first moment [17]. Table 2 lists a statistical summary of the prognostic factors of the A arm before and after balancing as well as the target aggregate data of the $\mathrm{B}$ arm using one of the repetitions as an example. Three analytic strategies were evaluated for unanchored MAIC in the first scenario. The first strategy was an unweighted analysis ignoring the unbalanced prognostic factors across trials. In the second strategy, all prognostic variables were included when conducting entropy balancing to create weight. In the third strategy, the indictors for menopausal status and tumor grades were omitted in the creation of weight.

In the second scenario, four analytic strategies were evaluated. Similar to the first scenario, the first and 
Table 2 Example of entropy balancing

\begin{tabular}{|c|c|c|c|}
\hline & \multicolumn{2}{|l|}{ A arm } & \multirow{2}{*}{$\begin{array}{l}\text { B arm } \\
\text { Aggregate data (target of balance) }\end{array}$} \\
\hline & Pre-balance & Post-balance & \\
\hline Mean age (years) & 52.55 & 56.14 & 56.14 \\
\hline Variance of age & 125.3 & 143.0 & 142.9 \\
\hline Postmenopausal (\%) & 40.00 & 52.12 & 52.13 \\
\hline Tumor grade $2^{\mathrm{a}}(\%)$ & 65.20 & 72.86 & 72.88 \\
\hline Tumor grade $3^{\mathrm{a}}(\%)$ & 14.40 & 18.37 & 18.38 \\
\hline
\end{tabular}

${ }^{a}$ Tumor grade 1 was a left-out reference group

second strategies were an unweighted analysis and a weighted analysis using all prognostic factors, respectively. The third strategy was a weighted analysis omitting the effect modifier (menopausal status) in the creation of weight, while the fourth strategy further dropped tumor grade indicators from the balanced variable list.In all analyses, Cox regressions were used to estimate the HRs, and the comparison of strategies was based on logHRs.

\section{Performance of unanchored MAIC}

To quantify the performance of unanchored MAIC in the analysis of time-to-event outcomes, the bias, the Monte-Carlo variance (MCV), the mean squared error (MSE), and the percentages of CI coverage of the estimates were evaluated for each strategy. Among these, MCV is the squared of the empirical standard errors [18], which is a measure of the efficiency of the estimator. The bias was calculated as

$$
\frac{1}{\mathrm{~N}} \sum_{j=1}^{N}\left(\log \widehat{\mathrm{HR}}_{\mathrm{j}}-(-0.5)\right),
$$

the MCV was calculated as

$$
\frac{1}{\mathrm{~N}-1} \sum_{j=1}^{N}\left(\log \widehat{\mathrm{HR}}_{\mathrm{j}}-\log \overline{\mathrm{HR}}\right)^{2}
$$

and the MSE was calculated as

$$
\frac{1}{\mathrm{~N}} \sum_{j=1}^{N}\left(\log \widehat{\mathrm{HR}}_{\mathrm{j}}-(-0.5)\right)^{2}
$$

where $\widehat{H R}_{j}$ is the estimated $\mathrm{HR}$ from the $\mathrm{jth}$ repetition in each scenario and $\overline{\mathrm{HR}}$ is the mean of $\widehat{\mathrm{HR}}_{\mathrm{j}}$ over $\mathrm{N}$ repetitions.

In addition to these quantities, the effective sample size (ESS) was also calculated [19]. Although not an indicator of the performance of MAIC estimates, ESS was informative in that its value should be close to the true sample size of the B arm when the characteristics of the two arms were balanced without having to rely on extreme weights [3]. A flowchart of the overall process of simulation, analysis, and comparison is illustrated in Fig.
S1. Engauge Digitizer 10.11 [20] was used to digitize the $\mathrm{K}-\mathrm{M}$ curves (screenshots of digitizing and exporting displayed in Figs. S2, S3, S4). Reconstruction of RIKM was implemented using Stata ipdfc routine and all statistical analyses were conducted using Stata 14 (StataCorp LLC, College Station, Texas, the United States of America) [10].

\section{Using the bias factor to estimate the boundary of the true effect}

For an exposure E, an unmeasured dichotomous confounder $\mathrm{U}$, and an outcome D, VanderWeele et al. has shown that

$$
\frac{H R_{\text {obs }}}{H R_{\text {true }}} \leq \text { bias factor }
$$

where $H R_{o b s}$ is the observed effect and $H R_{\text {true }}$ is the true effect. The bias factor is calculated as

$$
\left(H R_{U D} \times R R_{E U}\right) /\left(H R_{U D}+R R_{E U}-1\right)
$$

where $H R_{U D}$ is the maximal possible effect of $\mathrm{U}$ on $\mathrm{D}$ and $R R_{E U}$ is the risk ratio of $\mathrm{U}=1$ of the exposed group to the non-exposed group [21, 22]. As such, the inequality $H R_{\text {true }} \geq \frac{H R_{\text {obs }}}{\text { bias factor }}$ sL suggests that $H R_{\text {true }}$ should not be smaller than $\frac{L L \widehat{H R}}{\text { bias factor }}$ in $95 \%$ of the repetitions in which $L L_{\widehat{H R}}$ is the lower limit of the 95\% CI. If so, the strongest plausible effect can be estimated using $H R_{U D}$ and $R R_{E U}$. The former can be estimated using the IPD of the trial that the researchers can access, the latter can be based on assumptions or external sources. We calculated $\frac{\widehat{H R}}{\text { bias factor }}$ and $\frac{L L \widehat{H R}}{\text { bias factor }}$ for the two scenarios by setting menopausal status as $\mathrm{U}$, following which we summarized the mean bias of $\log \frac{\widehat{H R}}{\text { bias factor }}$ and the percentages of the repetitions in which $\frac{L L \widehat{H R}}{\text { bias factor }}$ was smaller than the true value. By the set-up of the data simulation, the bias factor was 1.10 and 1.05 in the two scenarios, respectively (the calculation was illustrated in online supplementary materials part II). 


\section{Results}

The results of comparing the raw data and RIKM of the $B$ arms as a validation of the reconstruction method are listed in Table 3. A graphical example of a raw survival curve and the counterpart using the digitization and reconstruction method is presented in Fig. S5. The mean HRs of the recovered indicator in the first and the second scenarios were correspondingly 0.959 and 0.960 , whereas the mean HRs of time-varying effect in both scenarios were 1.00. Also, the percentages of repetitions in which the $95 \%$ CI covered one were $100 \%$ in both scenarios.

The results of the performance evaluation of unanchored MAIC for survival outcomes in scenario 1 are presented in Table 4. In the first scenario, which did not involve any effect modifiers, the bias of the logHRs using the unweighted Cox regressions was 0.164. By contrast, the bias of the weighted Cox regressions that used all prognostic factors in entropy balancing was substantially smaller at 0.027 . Although less than the unweighted analyses, the bias of the weighted analyses when the indicators of menopausal status and tumor grades were dropped from entropy balancing was 0.114 . More, the MCV of the estimates was the same across all analytic strategies, which was 0.002 . Even more, the MSEs of the three analytic strategies were $0.029,0.003$ and 0.015 , respectively. Finally, the percentages of repetitions in which the $95 \%$ CI covered the true value were 11.2, 93.8 and $39.1 \%$ for the unweighted, fully weighted, and partially weighted strategies. None of the coverage reached the expected $95 \%$ although the fully weighted approach reached a close approximation.

The performance evaluation results related to scenario 2 are listed in Table 5. In the second scenario, the unweighted analysis had a bias of 0.173 . The fully weighted analyses had a bias of 0.035 . In addition, the bias of the weighted analyses omitting the effect modifier was 0.079 . More, the weighted analyses omitting both the indicator of menopausal status and the indicators of tumor grades had a bias of 0.122. The MCV of the unweighted estimator and the weighted approach omitting both the indicator of menopausal status and the indicators of tumor grades was 0.002 whereas that of the other two analytic strategies was 0.003 . The MSEs of these four analytic strategies were $0.032,0.004,0.009$ and 0.017 , respectively. The percentages of repetitions in which the 95\% CI covered the true value were 7.7, 89.9, 68.7 and $34.1 \%$ for the four strategies correspondingly. Similar to scenario 1 , the fully weighted approach in scenario 2 was the closest to the threshold of $95 \%$ but had an even greater shortage in coverage compared with scenario 1.

By the study design, the ESS of the fully weighted approach was the same in the two scenarios. Specifically, the ESS was 791 when all covariates were balanced, which was close to the true sample size of the B arm. As expected, the ESS was greater when the list of covariates for balancing was shorter.

The performance of adjustment methods using bias factors are presented in Table 6 . The mean bias of the bias factor-adjusted HRs in the log scale was -0.025 and 0.030 in the two scenarios, respectively. The magnitude of bias of the adjusted HRs were comparable to that of the fully weighted approaches in both scenarios. The corresponding percentages of repetitions of which the true value was not less than the adjusted lower limit (LL) were 93.3 and $91.8 \%$, respectively. These percentages were close to but did not reach the expectation of $95 \%$.

\section{Discussion}

In the present analysis, we examined the performance of alternative unanchored MAIC approaches to analyze time-to-event outcomes under the scenarios with and without an effect modifier. The results contribute to the information basis for the appropriate use of unanchored MAIC.

With a simulation, the present study confirmed that RIKM using the method proposed by Guyot et al. may sufficiently represent the raw time-to-event data [9]. This finding has two practical implications. First, secondary analyses using reconstructed IPD is a viable solution when raw data cannot be accessed. Second, and in a reversed perspective, studies on properties of methods related to reconstructed IPD may rely on simulated raw data instead of reconstructed data.

Our findings also revealed several important properties of unanchored MAIC. First and foremost, unanchored MAIC does have the potential to generate unbiased estimates when used to analyze time-to-event outcomes if all factors that impact either the outcome or the treatment effect are captured. That is, not only the effect

Table 3 Agreement between the raw data and the reconstructed data of the B arms

\begin{tabular}{lll}
\hline & Scenario 1 & Scenario $\mathbf{2}$ \\
\hline HR of recovered vs. raw indicator & 0.959 & 0.960 \\
Coverage of the $95 \% \mathrm{Cl}$ of the HR of indicator (\% of repetitions) & 100 & 100 \\
HR of time-varying effect & 1.000 & 1.000 \\
Coverage of the $95 \% \mathrm{Cl}$ of time-varying effect (\% of repetitions) & 100 & 100 \\
\hline
\end{tabular}

Abbreviations: HR Hazard ratio; Cl 95\% Confidence interval 
Table 4 Estimates of log hazard ratio in scenario 1 (without effect modifiers)

\begin{tabular}{llll}
\hline Statistical properties & Unweighted estimate & Weighted estimate \\
\cline { 3 - 4 } & & All variables included in weight & Omitted menopausal status and tumor grade from weight \\
\hline Bias & 0.164 & 0.027 & 0.114 \\
MCV & 0.002 & 0.002 & 0.002 \\
MSE & 0.029 & 0.003 & 0.015 \\
Cl coverage (\%) & 11.2 & 93.8 & 39.1 \\
mean ESS & NA & 791 & 888 \\
\hline
\end{tabular}

Abbreviation: MCV Monte-Carlo variance; MSE Mean squared error; Cl 95\% Confidence interval; ESS Effective sample size

modifiers but also the non-effect-modifying prognostic factors have to be balanced. Consistent with intuition, dropping some of the prognostic factors in balancing causes greater bias than balancing with full information but less bias than the unweighted approach. Second, and unlike the anchored counterpart, prognostic factors are important in unanchored MAIC analysis of time-toevent outcomes even though not being effect modifiers at the same time. In our simulation analyses, bias arose when not balancing on the prognostic factors even when there were no effect modifiers. Also, omitting the effect modifier which was also a prognostic factor led to nontrivial bias in the scenario of having an effect modifier. On top of bias, the confidence interval estimates of this approach was far from acceptable. As such, the results of the second scenario indicate that balancing both prognostic factors and effect modifiers is crucial in unanchored MAIC on time-to-event outcomes. Third, there may be a trade-off between bias and precision, yet the fully weighted approach constantly outperformed other approaches when MSE was used to evaluate the methods whereas the unweighted approach consistently ranked the worst. Therefore, the benefit of reducing bias with the fully weighted approach outweigh the precision loss in the setting of the present simulation analysis. Fourth, the uncertainty of unanchored MAIC may be biased or underestimated even when the bias of the relative effect is not a prominent problem because the coverage of the CIs never reached $95 \%$ across all strategies. This property of MAIC has not been spotted in literature previously and should be discussed in future applications of unanchored MAIC on time-to-event outcomes. The reasons of this property could be multifaceted. The loss of information in the data reconstruction step, although minuscule, may have contributed to this. More, entropy balancing followed by a Cox regression may not have fully accounted for bias. The compound of these sources of uncertainty may result in the imperfect confidence interval estimates.

In addition, we proposed an approach to estimate the boundary of the true effect when unanchored MAIC on time-to-event outcomes is likely biased due to omitted covariates. Simulation results showed that the proposed method was imperfect but were not necessarily unusable. This approach involves calculating the bias factor, which requires knowledge or assumptions of the extent of omitted variable unbalance $\left(R R_{E U}\right)$. In practice, the extent of omitted variable unbalance may be unknown in most situations. Possible solutions including using external data to estimate a plausible value of $R R_{E U}$ or calculating the adjusted HRs and the boundaries by toggling $R R_{E U}$ over a possible range. For example, if the percentages of post-menopausal individuals among breast cancer patients range from 35 to $65 \%$ across different trials and observational studies, then the range can be used to obtain the extremes of $R R_{E U}$ estimates, and, for that matter, the boundaries of bias factoradjusted treatment effect estimates. Of note, when the treatment effect is suspected to be overestimated rather than underestimated, the upper limit should be multiplied by the bias factor to estimate the boundary.

Table 5 Estimates of log hazard ratio in scenario 2 (with an effect modifier)

\begin{tabular}{|c|c|c|c|c|}
\hline \multirow{2}{*}{$\begin{array}{l}\text { Statistical } \\
\text { properties }\end{array}$} & \multirow{2}{*}{$\begin{array}{l}\text { Unweighted } \\
\text { estimate }\end{array}$} & \multicolumn{3}{|l|}{ Weighted estimate } \\
\hline & & $\begin{array}{l}\text { All variables } \\
\text { included in weight }\end{array}$ & $\begin{array}{l}\text { Omitted the effect modifier } \\
\text { (menopausal status) from weight }\end{array}$ & $\begin{array}{l}\text { Omitted the effect modifier (menopausal status) } \\
\text { and tumor grade from weight }\end{array}$ \\
\hline Bias & 0.173 & 0.035 & 0.079 & 0.122 \\
\hline MCV & 0.002 & 0.003 & 0.003 & 0.002 \\
\hline MSE & 0.032 & 0.004 & 0.009 & 0.017 \\
\hline $\begin{array}{l}\mathrm{Cl} \text { coverage } \\
(\%)\end{array}$ & 7.7 & 89.9 & 68.7 & 34.1 \\
\hline mean ESS & NA & 791 & 827 & 888 \\
\hline
\end{tabular}

Abbreviation: MCV Monte-Carlo variance; MSE Mean squared error; Cl 95\% Confidence interval; ESS Effective sample size 
Table 6 Bias factor-adjusted HR and lower limits of the 95\% Cl of HR using menopausal status as an omitted variable

\begin{tabular}{lll}
\hline & Scenario $\mathbf{1}$ & Scenario $\mathbf{2}$ \\
\hline Mean bias of adjusted HR in the log scale & -0.025 & 0.030 \\
Adjusted LL of the Cl of HR $\leq$ true effect (\% of repetitions) & 93.3 & 91.8 \\
\hline
\end{tabular}

Abbreviations: HR, hazard ratio; Cl 95\% Confidence interval; LL Lower limit

Several limitations should be noted when interpreting the results. First, we only used Weibull distribution to simulate the data sets. The data generation process in the real world may not necessarily approximate a Weibull distribution. Especially, survival curves in oncology are sometimes characterized by a high death rate due to nonresponse at the beginning or a plateau at the tail due to cure [23], which may not be sufficiently represented by single-index survival functions. As such, the generalizability of our findings is possibly limited due to the specific situations. Second, only 1000 repetitions were conducted for each scenario of data generation due to the labor-demanding process of manually completing part of the K-M curve digitization. Although the number of repetitions matches that of a previous simulation study in the realm of MAIC [5], the possibility of insufficient repetitions to reveal the properties could not be fully ruled out. Third, the same specification of shape and scale parameters of the Weibull distribution was used in the simulation of both A and B arms, which may be reasonable if the populations are adequately homogenous across trials. However, the scenarios of different underlying survival distributions across trials were not probed. Such complexity almost infinitely complicates the examination and discussion of any evidence synthesis methods. Fourth, the scenarios we explored were not exhaustive. For example, the coefficient specifications of covariates, the differences in the prognostic factors across trials, and the treatment effect were not extensively varied to examine the performance of unanchored MAIC under other scenarios. Such practice was largely hampered by the hefty manual work required to digitize the graphs. A byproduct of the limited number of scenarios for characteristic differences was that the impact of extreme weights due to larger differences in the IPD and the aggregate data could not be investigated, which was also reflected by the ESS of the fully weighted approach. Finally, censoring was simulated using a uniform distribution that was unrelated to the treatment, the effect modifier, and the prognostic factors. How non-random censoring impacts the performance of unanchored MAIC and MAIC in general in the analysis of time-to-event outcomes should be investigated in future.

\section{Conclusions}

Reconstructed IPD from digitized K-M curves may sufficiently represent the raw time-to-event data. Also, unanchored MAIC may be used in the analysis of timeto-event outcomes across single-arm trials. However, it should be used with caution of unmeasured prognostic factors and effect modifiers as well as suboptimal CIs. More, the bias factor-adjusted estimate can be used as an approximation of the boundary of the true effect at the presence of omitted variables.

\section{Supplementary information}

Supplementary information accompanies this paper at https://doi.org/10. 1186/s12874-020-01124-6.

\section{Additional file 1.}

\section{Abbreviations}

Cl: Confidence interval; HR: Hazard ratio; IPD: Individual-level patient data; KM: Kaplan-meier; LL: Lower limit; MAIC: Matching-adjusted indirect comparison; MCV: Monte-carlo variance; MSE: Mean squared error; NMA: Network meta-analyses; $R_{E U}$ : The extent of omitted variable unbalance; RFS: Recurrence-free survival time; RIKM: Reconstructed IPD using digitized K-M curves; USFDA: US food and drug administration

\section{Acknowledgements}

Not applicable.

\section{Authors' contributions}

YJ contributed to the conceptualization, methodology, software, formal analysis, data interpretation, and manuscript drafting of the study. WN contributed to the validation of the analysis, data interpretation, and review and editing of the manuscript. Both authors read and approved the final manuscript.

\section{Funding}

This research did not receive any specific grant from funding agencies in the public, commercial, or not-for-profit sectors.

\section{Availability of data and materials}

The study did not collect primary data. Program code files used for data simulation and analyses in the submitted work can be accessed at https:// doi.org/10.17632/6dvrxd7xpn.2.

\section{Ethics approval and consent to participate}

Not applicable.

Consent for publication

Not applicable.

\section{Competing interests}

The authors declare that they have no competing interests.

\section{Author details}

${ }^{1}$ School of Public Health (Shenzhen), Sun Yat-sen University, Room 215, Mingde Garden \#6, 132 East Outer Ring Road, Pan-yu District, Guangzhou, Guangdong, China. ${ }^{2}$ Department of Pharmaceutical and Health Economics, University of Southern California, 635 Downey Way, Verna \& Peter Dauterive Hall (VPD) Suite 210, Los Angeles, CA 90089-3333, USA. 
Received: 24 May 2020 Accepted: 18 September 2020

Published online: 29 September 2020

\section{References}

1. Phillippo DM, Ades AE, Dias S, Palmer S, Abrams KR, Welton NJ. Methods for population-adjusted indirect comparisons in health technology appraisal. Med Decis Mak. 2018:38(2):200-11.

2. Phillippo DM, Dias S, Ades AE, Belger M, Brnabic A, Schacht A et al. Multilevel network meta-regression for population-adjusted treatment comparisons. J R Stat Soc Ser A. 2020;183(3):1189-1210. https://doi.org/10. 1111/rssa.12579.

3. Signorovitch JE, Sikirica V, Erder MH, Xie J, Lu M, Hodgkins PS, et al. Matching-adjusted indirect comparisons: a new tool for timely comparative effectiveness research. Value Health. 2012;15(6):940-7.

4. Signorovitch J, Erder MH, Xie J, Sikirica V, Lu M, Hodgkins PS, et al. Comparative effectiveness research using matching-adjusted indirect comparison: an application to treatment with guanfacine extended release or atomoxetine in children with attention-deficit/hyperactivity disorder and comorbid oppositional defiant disorder. Pharmacoepidemiol Drug Saf. 2012;21:130-7.

5. Petto H, Kadziola Z, Brnabic A, Saure D, Belger M. Alternative weighting approaches for anchored matching-adjusted indirect comparisons via a common comparator. Value Health. 2019;22(1):85-91.

6. Phillippo D, Ades T, Dias S, Palmer S, Abrams KR, Welton N. NICE DSU technical support document 18: methods for population-adjusted indirect comparisons in submissions to NICE. 2016.

7. Committee PBA. Guidelines for preparing submissions to the pharmaceutical benefits advisory Committee (version 5.0. 2016). Canberra: Pharmaceutical Benefits Advisory Committee; 2016.

8. Malangone E, Sherman S. Matching-adjusted indirect comparison analysis using common SAS ${ }^{\oplus}$ 9.2: procedures. 2016. https://support.sas.com/ resources/papers/proceedings11/228-2011.pdf. Accessed Aug 182017.

9. Guyot P, Ades AE, Ouwens MJ, Welton N. Enhanced secondary analysis of survival data: reconstructing the data from published Kaplan-Meier survival curves. BMC Med Res Methodol. 2012;12(1):9.

10. Wei $Y$, Royston P. Reconstructing time-to-event data from published KaplanMeier curves. Stata J. 2017;17(4):786-802.

11. Ishak KJ, Rael M, Hicks M, Mittal S, Eatock M, Valle JW. Relative effectiveness of sunitinib versus everolimus in advanced pancreatic neuroendocrine tumors: an updated matching-adjusted indirect comparison. J Comp Eff Res. 2018;7(10):947-58. https://doi.org/10.2217/cer-2018-0020. Epub 2018 Aug 31. PMID: 30168349.

12. Sherman S, Amzal B, Calvo E, Wang X, Park J, Liu Z, et al. An indirect comparison of Everolimus versus Axitinib in US patients with advanced renal cell carcinoma in whom prior Sunitinib therapy failed. Clin Ther. 2015;37(11):2552-9.

13. Atkins MB, Tarhini A, Rael M, Gupte-Singh $K$, O'Brien E, Ritchings $C$, et al. Comparative efficacy of combination immunotherapy and targeted therapy in the treatment of BRAF-mutant advanced melanoma: a matching-adjusted indirect comparison. Immunotherapy. 2019;11. https:/doi.org/10.2217/imt-2018-0208.

14. U.S. Food and Drug Administration. Hematology/Oncology (Cancer) Approvals \& Safety Notifications. 2019. https://www.fda.gov/drugs/ resources-information-approved-drugs/hematologyoncology-cancerapprovals-safety-notifications. Accessed Mar 142019.

15. Hainmueller J. Entropy balancing for causal effects: a multivariate reweighting method to produce balanced samples in observational studies. Polit Anal. 2011;20. https://doi.org/10.2139/ssrn.1904869.

16. Hainmueller J, Xu Y. Ebalance: a stata package for entropy balancing. J Stat Softw. 2013;54(7):18. https://doi.org/10.18637/jss.v054.i07.

17. Greene WH. Econometric analysis. Boston: Prentice Hall Inc.; 2012.

18. Morris TP, White IR, Crowther MJ. Using simulation studies to evaluate statistical methods. Stat Med. 2019;38(11):2074-102. https://doi.org/10.1002/sim.8086.

19. Kish L. Survey sampling. vol 04; HN29, K5.: 1965.

20. Mitchell M, Muftakhidinov B, Winchen T, Jędrzejewski-Szmek Z, Trande A, Weingrill J et al. Engauge Digitizer Software. 2019. http://markummitchell. github.io/engauge-digitizer. Accessed Apr 272019.

21. VanderWeele T, Ding $P$, Mathur M. Technical considerations in the use of the E-value. J Causal Inference. 2019. https://doi.org/10.1515/jci-2018-0007.

22. VanderWeele TJ, Ding P. Sensitivity analysis in observational research: introducing the E-value. Ann Intern Med. 2017;167(4):268-74. https://doi.org/10.7326/m16-2607.

23. Farewell VT. Mixture models in survival analysis: Are they worth the risk? 1986;14(3):257-62. doi:https://doi.org/10.2307/3314804.

\section{Publisher's Note}

Springer Nature remains neutral with regard to jurisdictional claims in published maps and institutional affiliations.

\section{Ready to submit your research? Choose BMC and benefit from:}

- fast, convenient online submission

- thorough peer review by experienced researchers in your field

- rapid publication on acceptance

- support for research data, including large and complex data types

- gold Open Access which fosters wider collaboration and increased citations

- maximum visibility for your research: over $100 \mathrm{M}$ website views per year

At $\mathrm{BMC}$, research is always in progress.

Learn more biomedcentral.com/submissions 